\title{
The Concept of IMPRESSION: An Interactive Instruction System and Its Practice for Real-Time Distance Lessons between U.S. and Japan
}

\author{
Takashi Mitsuishi ${ }^{1}$, Fumiko Konno ${ }^{1}$, Yuki Higuchi ${ }^{2}$, and Kentaro Go ${ }^{3}$ \\ ${ }^{1}$ Graduate School of Educational Informatics, Tohoku University \\ Kawauchi 27-1, Aoba-ku, Sendai, Japan \\ \{takashi, fumiko\}@ei.tohoku.ac.jp \\ ${ }^{2}$ PRO \& BSC Corporation \\ Honcho 2-1-8, Aoba-ku, Sendai, Japan \\ hyukix@gmail.com \\ ${ }^{3}$ Interdisciplinary Graduate School of Medicine and Engineering,University of Yamanashi \\ Takeda 4-4-37, Kofu, Japan \\ go@yamanashi.ac.jp
}

\begin{abstract}
In order to perform flexible and effective lesson, we proposed "Double Loop Instructional Design Process Model" and developed an interactive instruction system; IMPRESSION based on the proposed model. In this paper, we show the concept of IMPRESSION and also describe the realtime distance lessons we conducted continuously in the first semester of 2007 with IMPRESSION and Skype by connecting between Mountain View, CA, U.S. and Tohoku University, Sendai, Japan via the Internet. As a result of these lessons, we confirmed that although we had a little time lag and some QoS problems of video stream caused by narrow bandwidth and very long distance, we could perform flexible and effective real-time distance lessons by using IMPRESSION and a videoconference system like ordinary lessons such as chalk and talk lessons in a classroom. However, we also found some points to be improved on for continuous use of IMPRESSION.
\end{abstract}

Keywords: real-time distance lesson, interactive electronic whiteboard system, multimedia materials, instructional design process model.

\section{Introduction}

According to the growth of information processing and communication technologies in recent years, there exist so many studies on distance education and utilization of multimedia materials [1][2][3][4][5]. However, existing systems allow us to communicate by only videoconference systems or to show additional materials in web pages or slides and write annotation on it. Moreover, when we use very long distance network such as between U.S. and Japan, narrow bandwidth and time lag make us difficult to communicate interactively with complex multimedia materials.

In order to cope with these problems and perform flexible and effective lessons, we proposed Double Loop Instructional Design Process Model which allows us to 
modify lesson plan immediately based on formative evaluations in a lesson, and developed an interactive instruction system; IMPRESSION[6]. In this paper, we show the concept of IMPRESSION and also describe the result of the real-time distance lessons we performed continuously for half a year by connecting between U.S. and Japan via the Internet with IMPRESSION and a videoconference system.

\section{Double Loop Model and IMPRESSION}

IMPRESSION is an instruction system for flexible and effective lesson with multimedia materials. It is a sort of sharable and interactive electronic whiteboard system based on Double Loop Instructional Design Process Model (Double Loop model) we proposed [7].

\subsection{Double Loop Model}

Double Loop model is an instructional design process model for designing interactive lessons by using information technologies and it forms double loop shown in Fig 1. The outer loop defines the activities of designing a lesson, and is consists of (1) Plan, (2) Apply, and (3) Evaluate phases. The inner loop of Apply phase defines the activities in a lesson, and is consists of (1) Implement, (2) Check, and (3) Modify phases.

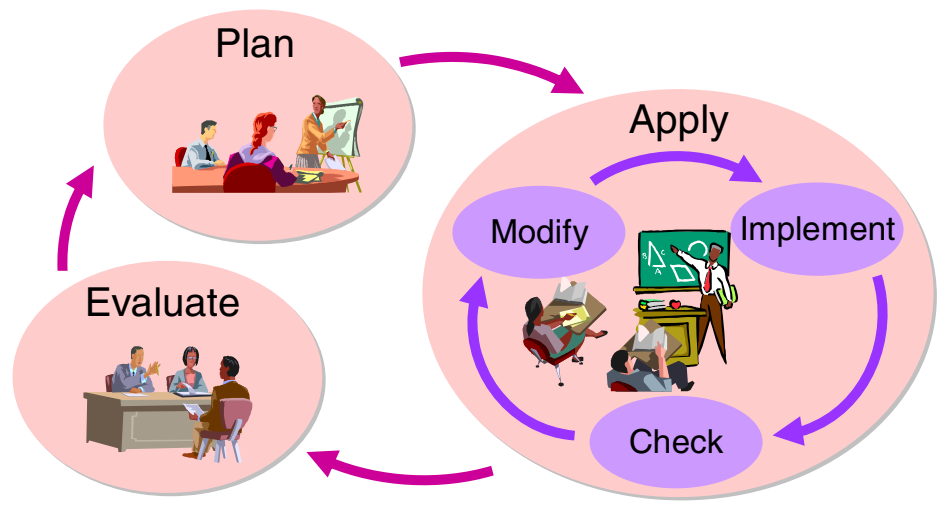

Fig. 1. Double Loop Instructional Design Process Model

As mentioned above, Double Loop model, differ from existing models, defines modification process of instructional design in a lesson in addition to instructional design process of a lesson. Thus, it clarifies interaction between a teacher and students, and allows us to cope with unexpected learners' reactions flexibly in a lesson. It improves effectiveness of a lesson. And we are also able to review processes of performed lessons after them and confirm the modifications from plans. It facilitates us to redesign next class with the results of actually performed lessons. 


\subsection{Facilities and Features of IMPRESSION}

IMPRESSION was designed and implemented as an instruction system based on our Double Loop model and allows us to perform activities in Apply phase. It consists of two types of terminals; a terminal for instructor and terminals for student, which are equipped with pen-based input device such as Tablet PC, and a relay server as shown in Fig. 2. We are able to apply this system to both in-class lesson and distance lesson. And it also allows us to utilize various multimedia data provided by web servers over the Internet as educational materials.

A teacher registers a set of multimedia data to use as educational materials in advance. During a lesson, he/she select ones at will from them, present them on a

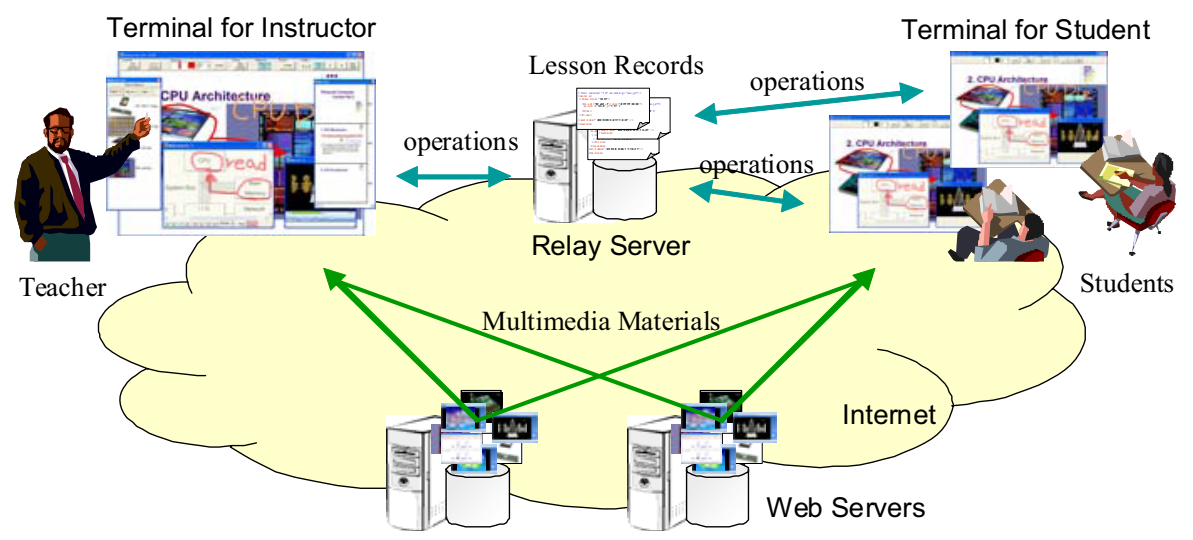

Fig. 2. System Construction of IMPRESSION

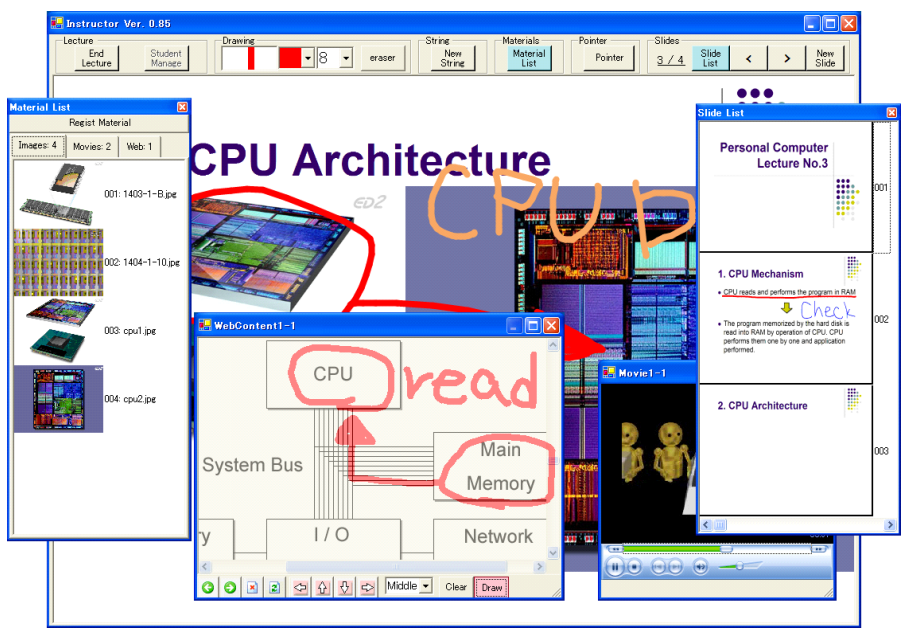

Fig. 3. A Screenshot of IMPRESSION terminal for instructor 
whiteboard, handle them (e.g. move, resize), and draw annotations on them by handwriting with an IMPRESSION terminal for instructor. Fig 3 shows a screenshot of the terminal. With these operations, it facilitates us to conduct interactive lessons according to reaction of students with various multimedia materials not only in a class but also a distance class. Thus, it makes possible to conduct flexible lessons based on formative evaluation which was defined as Apply phase of Double Loop model.

\section{Practice of Real-Time Distance Lessons between U.S. and Japan}

This time, we conducted a series of lessons as real-time distance lessons continuously every week in the first semester of 2007 with IMPRESSION and Skype by connecting between U.S. and Japan via the Internet.

\subsection{Content and Class Format of Practiced Lessons}

The series of lessons we conducted this time is a course named "Fundamental of IT Education B." This course has been opened at Graduate School of Educational Informatics, Tohoku University from 2002 and provided to graduate students who have not always studied computer science or engineering. The first author lectures on fundamental computer engineering and information technologies for education (e.g. logical operation, database, computer network, and so on) in this course.

This time, the first author stayed at California for a business in 2007, so we decided to practice distance lessons. We conducted fourteen lessons through 13:00 to 14:30 on every Tuesday of Japan Standard Time (through 21:00 to 22:30 on Monday of Pacific Daylight Time) from April to July. Participated students were five, and the second author, who is a doctoral candidate, also participated as a teaching assistant in order to help students and operate computer systems for IMPRESSION and a videoconference system.

So far, the teacher had been preparing a lecture note and providing it before each lesson, assigning homework after every lesson, marking it and giving feedback to it, and returning it with comment at a next lesson. Therefore, this time, we provide lecture notes, receive reports for assignment, and return marked reports through a LMS of ISTU[8]. But, TA marked reports and gave feedback to them at this moment.

\subsection{Implemented Environment and System Configuration}

Fig. 4 shows an implementation environment and a system configuration for the practiced distance lessons. This time, we connected between a room in an apartment house at Mountain View, CA, U.S., where the first author lived in, and a classroom at Tohoku University, Sendai, Japan, where students participated to lessons, via the Internet, and used Skype[9] for videoconference in addition to IMPRESSION as shared whiteboard.

At the teacher's room, we prepared a Table PC (Toshiba PORTEGE M400) for both IMPRESSION and Skype and connect it to the Internet via DSL. At Tohoku University, we prepared two Tablet PCs, one (Toshiba PORTEGE M400) with video projector is for IMPRESSION, and another (Toshiba Dynabook SS 3500) with 50in. 


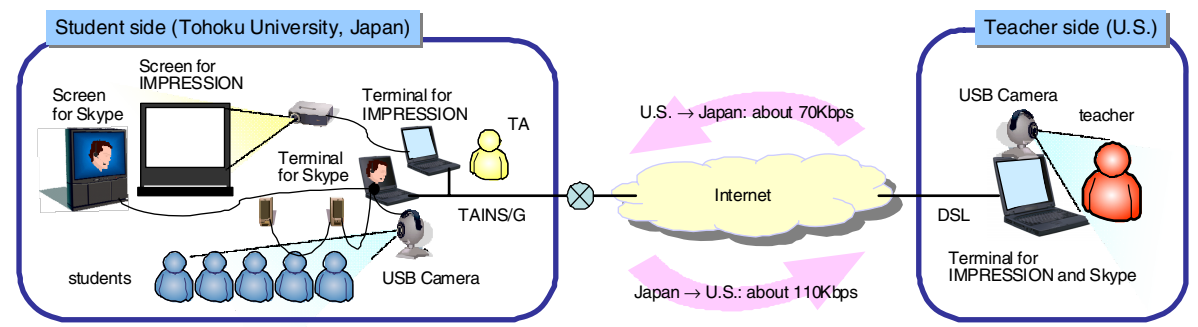

Fig. 4. Implemented Environment and System Configuration of Practiced Distance Lessons

wide screen plasma monitor is for videoconference by Skype. In order to capture the video, we prepared USB camera on both site. We also prepared speakers in the classroom. The teacher used Bluetooth wireless headset in order to prevent howling.

\subsection{Results of the Practices}

Fig. 5. (a) shows the front of the classroom where students participated and Fig. 5. (b) shows the terminals which TA operated in Japan. Fig. 5. (c) shows the teacher conducting a lesson by operating the terminal. Fig. 6 shows a screenshot of IMPRESSION in a practiced lesson.
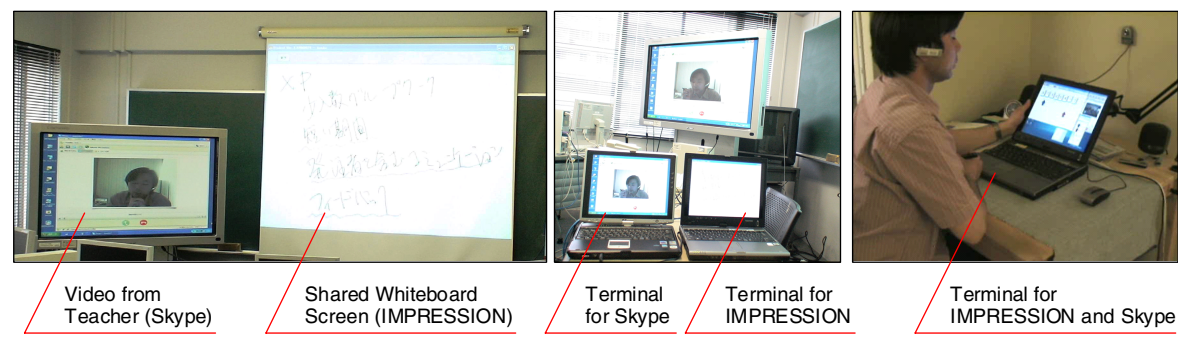

(a) Front of a Classroom in Japan

(b) Terminals in Japan

(c) A Terminal in U.S.

Fig. 5. Scenes of Practiced Distance Lessons

As results, although there were some problems that were necessary to reboot Skype for a few times because video streams froze and some audio communication failure occurred, IMPRESSION worked with no problem and we were able to conduct whole planned lessons almost well.

However it was not communication failure, there exited about 1 second time lag in audio and video streams which may be caused very long distance communication and encoding and decoding of streams. By restricted bandwidth, quality of video images was also not so clear. Therefore, we could not distinguish expression of each student very much even if he/she was closer to the camera when we wanted to see whole students. 


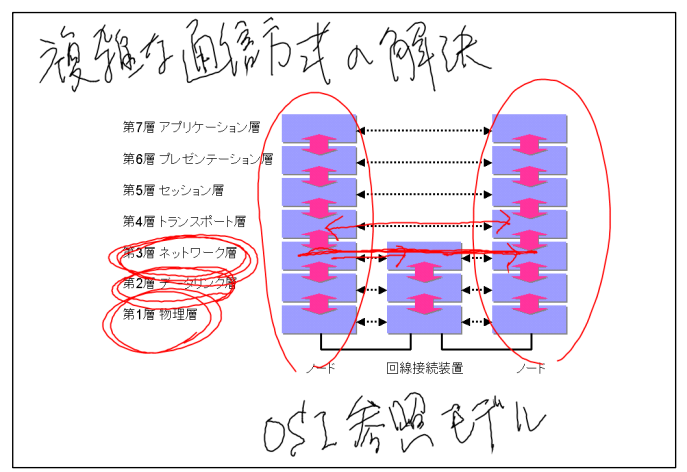

Fig. 6. A Screenshot of the Shared Whiteboard Screen in a Conducted lesson

\section{Evaluation}

Here we describe subjective evaluation by the teacher and also evaluation by participated students.

\subsection{Subjective Evaluation by the Teacher}

This time, although we had some problem on videoconference by Skype, we confirmed that we were able to conduct distance lessons with a videoconference system such as Skype and IMPRESSION as an interactive instruction system in combination. And it seems that they almost stand comparison with ordinary in-class chalk \& talk lessons from result of assignments and a term-end exam.

As we mentioned in the results, there exists time some lag in video streams and resolution of video images are restricted. We confirmed that these lacks of quality were affecting communication between the teacher and students. For example, even though sounds are clear enough, time lag upset timing to speak and prevent smooth communications. And the teacher sometimes felt difficulty to decide to put forward or explain again with different words because it was difficult to grasp whether students certainly understood or not from vague video images.

On the other hand, IMPRESSION worked well with its features although there are some restriction such as screen size and resolution in comparison with ordinary blackboards. For example, we are able to present any materials at will by registered them in advance, it reduced the load to write complex figures and tables in a lesson and the teacher could concentrate on explanations. IMPRESSION is able to go back to the screen in the past. With this feature, the teacher showed again the drawn screen after putting forwarding explanations. Such a progress is impossible in ordinary lesson with blackboard. And the more, by giving the right of operation to students, the teacher and students were able to interact with each other through the numbered cards on the screen of IMPRESSION when we discussed sorting algorithm. Therefore it facilitated the teacher to conduct not only flexible lessons which were the original objective of IMPRESSION but also smooth and effective lessons. 
However, we also found some problems to be solved as will be discussed later in this paper.

\subsection{Evaluation by Students}

In order to investigate the effectiveness of distance lessons with IMPRESSION and Skype, we conducted the survey with questionnaires to students [10]. Fig. 7 shows part of the result of questionnaires; from Q1 through Q7 on the systems and Q8 and Q9 on help and feedback by TA.

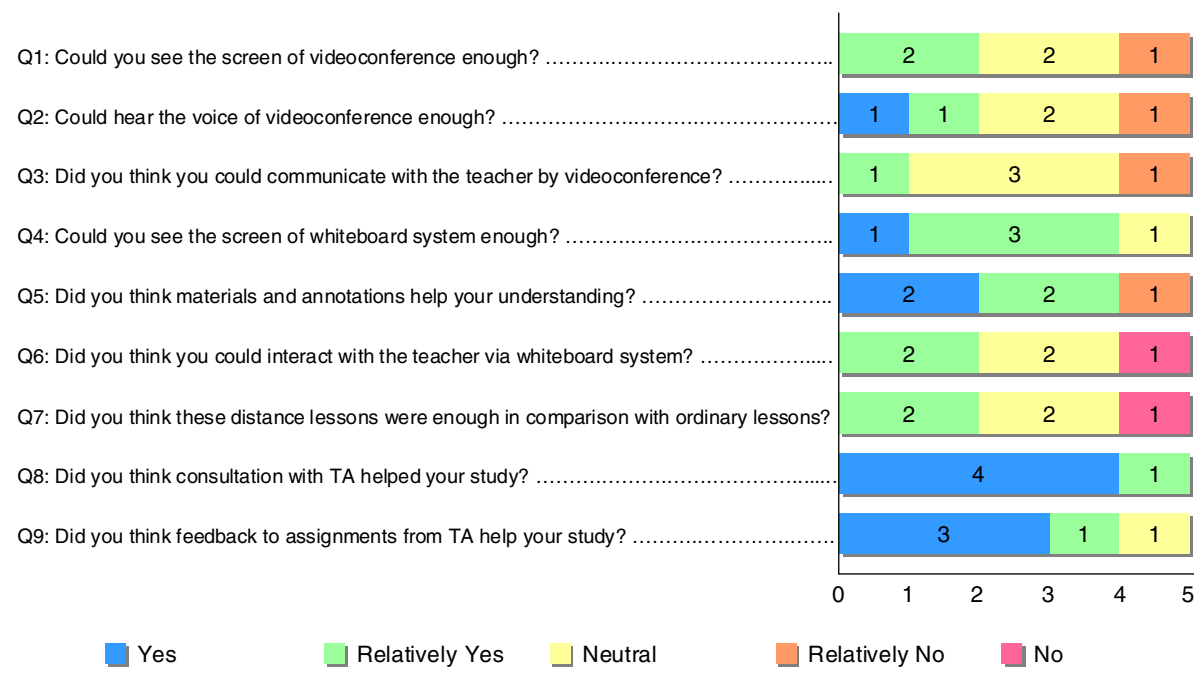

Fig. 7. Results of Questionnaire to Students

Table 1. Reasons why they felt not enough with these distance lessons

- Face to face lessons seem better than distance lessons.

- It seems a little difficult to communicate with the teacher.

- Sometimes voice had disconnected.

- Much better communication line seems necessary.

Although it doesn't have statistical meaning because the amount of participants were a very few at this moment, we can grasp a rough tendency. As a result, students felt that the systems were not enough but not too bad. About a question; "when you felt the systems are not enough, which are the problems of them or how to solve them?" they answered to this question as shown in Table 1.

From these opinions, most of the reasons why they did not feel enough or satisfied with the distance lessons are lack of stability of Skype causing freeze of video streams and audio communication failure, lack of quality of video images caused restricted bandwidth and time lags caused very long distance. 
As for drawing and presentation with IMPRESSION, although we had some negative answers to the question Q6: "Can you interact with the teacher via shared whiteboard screen?" we had relatively positive answers to the question Q5: "Is the explanation by drawing annotations and presenting materials on shared whiteboard screen helpful for your understanding?" It shows the effectiveness of features of IMPESSION for distance lessons. The reason of negative answers to Q6 might be assumed that we could prepare only one Table PC for IMPRESSION in a classroom, students should move to the terminal in order to operate it, and we could not interact at will via IMPRESSION.

And these are not about the systems such as IMPRESSION and Skype, the second author helped students, checked reports for assigned homework and gave feedback as TA. Q8 and Q9 were questions asking how affect these help and feedback by TA and the answers were almost very well. Thus, we could assume these help and feedback by TA made up for restrictions and problems in communication between the teacher and students.

\subsection{Confirmed Problems of IMPRESSION to Be Solved}

Although we find some restrictions of bandwidth and lack of stability of the videoconference system we used, these matters will be solved in the near future and we confirmed the effectiveness of IMPRESSION to sum up. However we also found some problems of IMPRESSION to be solved. The load to prepare materials and manage them is especially one of big problem of them.

This time was the first time to use IMPRESSION in this course and the teacher should prepare materials. So, the load to prepare was of course heavy. However, in addition to the load to prepare materials, we found following two problems; 1) it was burdensome to register materials to IMPRESSION or to delete registered materials from IMPRESSION, 2) it was difficult to find an aimed material when so many materials were registered.

Current IMPRESSION is just prototype to demonstrate interactive presentation of multimedia materials and has features for this purpose. Therefore it has only simple features and user interfaces to handle and manage materials. For example, it allows us to register materials one by one, but we are not able to register a set of materials at once. And it does not have a feature to sort out registered materials nor retrieve an aimed material by query. So, when we select a material to present in a lesson, we need to search the aimed material from all registered materials.

This time we conducted lessons continuously every week and materials for each lesson were different from others. So we should register a new set of materials every week. And, we assumed it was difficult to find an aimed material to present when so many materials were registered, we deleted materials which were registered for previous lessons. These were very burdensome tasks. Furthermore, even when we regard some materials were not necessary and deleted them, sometimes they became necessary in order to review previous lessons. It could be said as lack of reusability.

In order to solve these problems and facilitate to select an aimed material from many materials and to reuse registered materials in previous lessons, it is necessary not only to improve user interfaces but also to develop some features to sort and manage materials based on lesson plans. 


\section{Conclusion}

This time, we conducted real-time distance lessons continuously for half a year by connected between U.S. and Japan with Skype and IMPRESSION via the Internet. As a result, we confirmed that although there were several restrictions on videoconference via the Internet such as time lags and resolution of video images, we were able to conduct real-time distance lessons with a videoconference system and our IMPRESSION, which allows interactive presentation of multimedia materials and drawing annotations on a shared whiteboard screen among a teacher and students, and the effectiveness of such lessons in comparison with ordinary lessons in a classroom.

However, we also found the problems of IMPRESSION to be solved in order to conduct lessons continuously such as usability of user interfaces and features to handle and manage many materials. We are developing such features[11]. On the other hand, we will be able to record conducted lessons when we conduct them with a system like IMPRESSION, and it could be expected to create contents for review by students or to prepare data for reflection by the teacher[12][13][14][15][16]. We will solve the confirmed problems, improve our system, and propose novel educational environments as our future work.

Acknowledgments. A part of this work was supported by KAKENHI (16700550 and 20700631).

\section{References}

1. Yoshino, T., Inoue, Y., Yuizono, T., Munemori, J., Ito, S., Nagasawa, Y.: Development and Application of a Supporting System for Distance Learning Classroom Using Personal Computers via Internet. IPSJ Journal 39(10), 2788-2801 (1998)

2. Murakami, M., Yagi, K., Kakusho, K., Minoh, M.: Analysis of Evaluation Factor of Distance Learning System Focusing on Effect of Experience and Custom in Lecture (in Japanese). IEICE Transactions on Information and Systems J84-D-I(9), 1421-1430 (2001)

3. He, A., Kara, A., Cheng, Z., Go, K., Koyama, A., Huang, T., Imamiya, A.: RIDEE-SPS: A Presentation System for Realtime Interactive Distance Education Environment (in Japanese). IPSJ Journal 44(3), 700-708 (2003)

4. Hayashi, T., Watanabe, K., Otani, M., Tanaka, H., Okazaki, Y., Hayashida, Y., Kondo, H.: Design and Implementation of a Remote Lecture Based on Instruction with Blackboard Using High-Quality Media Devices and High Speed Information Network (in Japanese). Journal of Japanese Society for Information and Systems in Education 22(1), 3-14 (2005)

5. Matsuura, T., Maeda, K., Kohno, E., Kishida, T.: A Telepresentation System to Assist Presenting Rich Multimedia to Audiences in Remote Locations (in Japanese), Technical Report of JSiSE Chugoku branch 5(4), pp.16-19 (2005)

6. Higuchi, Y., Mitsuishi, T., Go, K.: An Interactive Multimedia Instructional System: IMPRESSION for Double Loop Instructional Design. IEICE Transaction on Information and Systems E89(6), 1870-1884 (2006)

7. Higuchi, Y., Konno, F., Mitsuishi, T., Go, K.: A Double Loop Instructional Design Process Model for Interactive Instructions. Japan journal of educational technology 3(4), 457-468 (2008) 
8. Mitsuishi, T., Kumai, M.: ISTU: Internet School of Tohoku University (in Japanese). Journal of IEICE 86(11), 816-820 (2003)

9. Skype web site, http://www. skype.com/

10. Mitsuishi, T., Konno, F.: A Report on Practice of Distance Class between Japan and U.S. with ISTU and Real-time Distance Education System (in Japanese). Annual report of ISTU 4, 9-21 (2006)

11. Suzuki, T., Konno, F., Ohkawa, Y., Mitsuishi, T.: Design and Implementation of a Lesson Plan Based Data Management Mechanism for Educational Materials in IMPRESSION. In: Proc. of 33th JSiSE Annual Conference, pp. 296-297 (2008)

12. Konno, F., Higuchi, Y., Mitsuishi, T.: A proposal of a methodology of supporting teacher reflection by presenting differences between planned and implemented actions. In: Proc. of AACE SITE 2007, pp. 1059-1064 (2007)

13. Konno, F., Higuchi, Y., Mitsuishi, T.: Teacher's Awareness in New Reflection Methodology Using Highlighted Process Displays. In: Proc. of AACE SIT 2008, pp. 2665-2673 (2008)

14. Kanno, Y., Konno, F., Ohkawa, Y., Hashimoto, K., Mitsuishi, T.: A Proposal of Teacher Reflection Supporting System Based on Observation of Reviewing Activities with Handouts and Video Records (in Japanese). Educational Informatics Research (7), 1-8 (2008)

15. Konno, F., Higuchi, Y., Mitsuishi, T.: A Study on a Teacher Reflection Method by Presenting Differences between Lesson Plan and Actual Instructions (in Japanese). Japan journal of educational technology, 32(4) (printing)

16. Konno, F., Kanno, Y., Mitsuishi, T.: Effects of Teacher Reflection with Highlighted Level Process Displays: Presenting Differences between Lesson Plan and Implemented Actions. In: Proc. of AACE SITE 2009, pp. 1236-1243 (2009) 